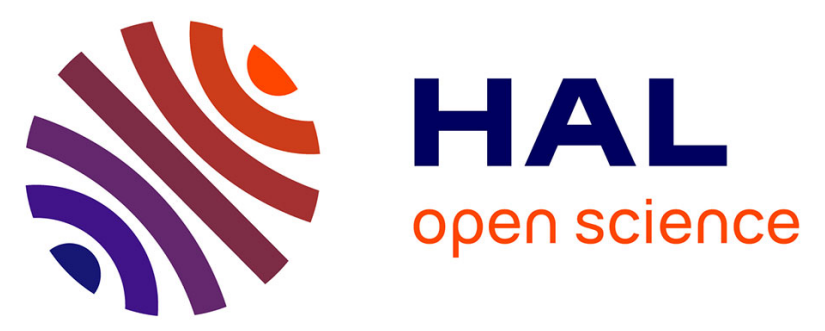

\title{
Potential of chipless authentication based on randomness inherent in fabrication process for $\mathrm{RF}$ and $\mathrm{THz}$
}

Zeshan Ali, Florent Bonnefoy, Romain Siragusa, Nicolas Barbot, David Hély, Etienne Perret, Maxime Bernier, Frédéric Garet

\section{- To cite this version:}

Zeshan Ali, Florent Bonnefoy, Romain Siragusa, Nicolas Barbot, David Hély, et al.. Potential of chipless authentication based on randomness inherent in fabrication process for RF and THz. 11th European Conference on Antennas and Propagation (EUCAP), Mar 2017, Paris, France. 10.23919/EuCAP.2017.7928647 . hal-01800579

\section{HAL Id: hal-01800579 \\ https://hal.science/hal-01800579}

Submitted on 13 Jan 2020

HAL is a multi-disciplinary open access archive for the deposit and dissemination of scientific research documents, whether they are published or not. The documents may come from teaching and research institutions in France or abroad, or from public or private research centers.
L'archive ouverte pluridisciplinaire HAL, est destinée au dépôt et à la diffusion de documents scientifiques de niveau recherche, publiés ou non, émanant des établissements d'enseignement et de recherche français ou étrangers, des laboratoires publics ou privés. 


\title{
Potential of Chipless Authentication Based on Randomness Inherent in Fabrication Process for RF and $\mathrm{THz}$
}

\author{
Zeshan Ali ${ }^{1}$, Florent Bonnefoy ${ }^{2}$, Romain Siragusa ${ }^{1}$, Nicolas Barbot ${ }^{1}$, David Hely ${ }^{1}$, Etienne Perret ${ }^{1,3}$, Maxime Bernier ${ }^{2}$, \\ and Frédéric Garet ${ }^{2}$ \\ ${ }^{1}$ University of Grenoble Alpes, LCIS, Valence, France, forename.lastname@ @ lcis.grenoble-inp.fr \\ ${ }^{2}$ University of Savoie Mont Blanc, IMEP-LAHC, Chambery, France, forename.lastname @ univ-savoie.fr \\ ${ }^{3}$ Institut Universitaire de France, Paris, France.
}

\begin{abstract}
In this paper, two chipless solutions dedicated to the authentication of manufactured products are proposed. One device is operating in the RF domain while the other used terahertz (THz) waves. The concept of chipless RFID is extended to authentication where each tag has to present a unique signature that can never be reproduced even if someone try to copy the tag. Both approaches use Electromagnetic (EM) wave as a tool for non-invasive and non-destructive authentication of items. The results show that it is possible to discriminate RF, respectively $\mathrm{THz}$, signatures obtained from devices whose differences of geometrical parameters are as small as tens of micrometers, respectively several micrometers in the THz domain.
\end{abstract}

Index Terms- authentication technology, anticounterfeiting, fabrication tolerance, microwave, randomness, RFID, terahertz.

\section{INTRODUCTION}

Anti-counterfeiting measures have always been desired due to exponential growth in grey market worldwide. At present, various anti-counterfeiting techniques are being utilized to limit this illegitimate practice of counterfeiting such as chemical technology (photochromic and thermochromic inks), biotechnology (DNA, enzymes, and antibodies), optical technology (holograms), and electronics (magnetic stripes, microchips, and physical unclonable function (PUF)).

Recently, chipless RF identification (RFID) is also emerging as one of the authentication and/or identification tool [1]-[4]. A hybrid system comprising optical and chipless RFID techniques is presented in [1], where each label has two distinctive signatures: optical and EM signature, which are used for identification and authentication, respectively. A new generation of terahertz (THz) chipless tags has been introduced in [2], where data encoding is done by using a multilayer structure along both of the surface and volume of the device. Conversely, a tagless RFID method to identify the electronic devices is presented in [3]. This method is based on capturing the EM signatures emitted by electronic devices and determining the uniqueness among these signatures by using cosine similarity function as a resemblance metric.
On the other hand, the randomness during the fabrication process can be exploited to generate unique response for each realization, which can be further employed for authentication [1]. In recent past an unclonable chipless RFID tag based on concentric ring slot resonators is presented in [4] on this subject. The process variations for trace width, air gap, substrate thickness, and dielectric constant of substrate have been considered and euclidean distance is used as a similarity measure to determine the uniqueness of the tags.

In this paper the extent of variability of EM signature due to modifications of dimension parameters inherent in realization process is discussed for two regimes of frequency spectrum: RF and THz. Section II is dedicated to evaluate the potential of doing authentication using chipless RFID technology, where a cavity resonator is used for measurement. Same demarche is presented in Section III where a device based on dielectric waveguide associated with a $1 \mathrm{D}$ diffraction grating is proposed for authentication in the $\mathrm{THz}$ domain.

\section{TOWARDS CHIPLESS RFID AUTHENTICATION}

\section{A. Principle}

A well-known C-folded scatterer has been discussed in [5], [6], where the response of such scatterer is controlled by two key dimension parameters: the spacing between two arms $g$ and the length $L$. The layout of the scatterer is shown in Fig. 1(a). The frequency of resonance $f_{r}$ can be calculated by following expression:

$$
f_{r}=\frac{c}{4(L+\Delta L) \sqrt{\varepsilon_{e f}}}
$$

where, $L$ is the length of each arm, $\varepsilon_{e f}$ is the effective permittivity of the substrate, and $\Delta L$ is a complementary length added due to the fringing fields.

The maximum level to which randomness can occur along the geometric dimensions during the fabrication process is approximately equal to the metal thickness .i.e. in our case $35 \mu \mathrm{m}$. For such $\mathrm{C}$-folded coplanar strip line structure [7] having dimensions $w=2 \mathrm{~mm}, g=0.8 \mathrm{~mm}$, $L+\Delta L=13.2 \mathrm{~mm}$, on Rogers RO 3003 substrate with $\varepsilon_{r}=3$, 
and height $h=0.75 \mathrm{~mm}$, it is found that $\varepsilon_{e f}=1.2795$ and $f_{r}=5.023 \mathrm{GHz}$. From (1) the maximum variation $(35 \mu \mathrm{m})$ merely along the length $L$ would cause a significant shift in frequency of resonance around $13.4 \mathrm{MHz}$. However, the prime focus of this study is to investigate the variations along other dimension parameters (except $L$ ) which have less effect on the resonance frequency. If we impose the assumed maximum variation $(35 \mu \mathrm{m})$ such that $g=0.8-0.035 \mathrm{~mm}$, $w=2+0.035 \mathrm{~mm}$ and leaving the $L+\Delta L$ fixed, it is found that that $\varepsilon_{e f}=1.2821$ and $f_{r}=5.018$. This shift of frequency is around $5 \mathrm{MHz}$ which requires a high sensitive measurement technique. As we can see in (1), the frequency of resonance of the tag is not directly linked to $g$ and $w$. For this reason cavity measurements are considered in this work which will be discussed in next section.

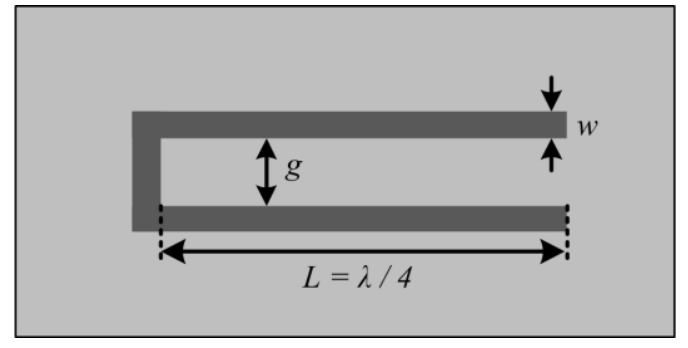

(a)
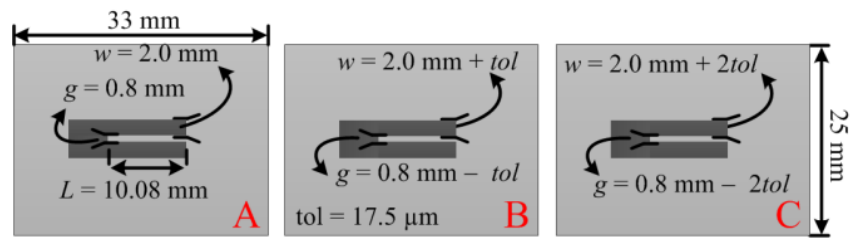

(b)

Fig. 1. Layouts of C-folded scatterer. (a) General layout. (b) Layouts of the fabricated tags.

\section{B. Measurement Setup}

Cavity resonators are well known for material characterization due to the shifts in the resonant modes happening while introduction of a foreign body. Similarly, It has been discussed in [8], [9], that introduction of a dipole in a cavity resonator causes a shift in the frequency of the resonant mode. This shift is even more significant when the resonant frequency of the cavity and of the device are closed form one to the other [9]. This property is very suitable to characterize the uniqueness of the $\mathrm{C}$-folded dipole subjected to random dimension modifications during realization process. To prove the concept, three tags [see Fig. 1(b)] are realized by imposing small geometric variations (multiple of fabrication tolerance $t o l=17.5 \mu \mathrm{m}$ ) to $g$ and $w$ while keeping $L$ same for all the tag.

The labeled A tag shown in Fig. 1(b) is having nominal geometric dimensions with a resonance frequency of $5 \mathrm{GHz}$. For the tag labeled as $\mathrm{B}$, the width of the trace $w$ is increased and the spacing between two arms $g$ is decreased by an imposed fabrication tolerance tol. This imposition for tag $\mathrm{C}$ is double as compared to tag $\mathrm{B}$. The entire remaining geometric dimensions are the same across these three tags. The overall size for all presented tags is same, that is, $33 \times 25 \mathrm{~mm}^{2}$.

In the measurement setup shown in Fig. 2, the DAMAKOS thin sheet tester cavity is used. This tester cavity is capable of generating various resonant modes in a frequency span ranging from $800 \mathrm{MHz}$ to $20 \mathrm{GHz}$. The external dimensions of this tester cavity are $45 \times 22 \times 5 \mathrm{~cm}^{3}$. The tags are placed in the middle of the cavity facing microstrip circuit towards the excitation ports with the help of a transparent plastic sheet having a holding window. This holding plastic sheet ensures the exact position for all the tags in different measurement trials. Transmission coefficient $\mathrm{S}_{21}$ is measured for each tag by twelve successive measurements, where each measurement is done by removing the tag and then putting back in place.

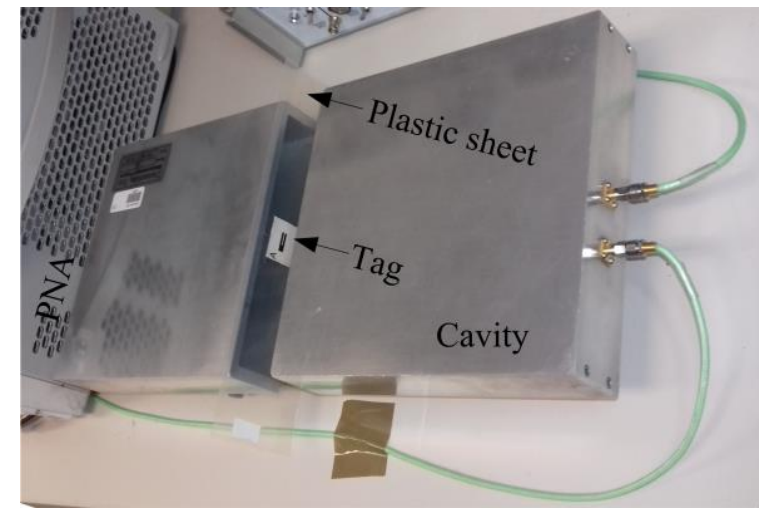

Fig. 2. The cavity measurement setup.

\section{Results}

Fig. 3 shows the $S_{21}$ parameter of the first measurement for the empty cavity, holding plastic sheet, and all of the three tags. In Fig. 3 the largest shift in frequency of resonant mode is due to the introduction of the plastic sheet, while the shift among the tags is less. This small shift is due to the minor geometric dimension impositions. The minor shifts of resonant modes close to resonance of fabricated tags are used to characterize the intra-tag and inter-tag uniqueness by using the cosine similarity function. Cosine similarity function is applied on $S_{21}$ parameter of twelve successive measurements of all three tags in the frequency domain.

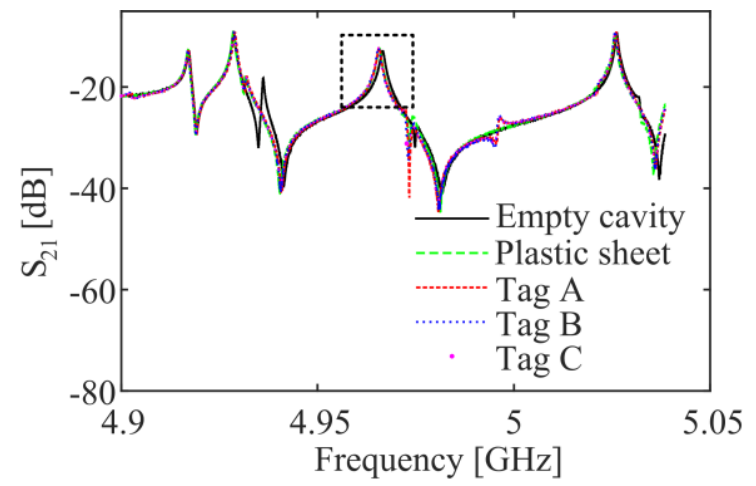

Fig. 3. Transmission coefficient of the first measurement for the empty cavity, holding plastic sheet alone, and all of the three tags. 
Table I summarizes the mean and standard deviation of cosine similarity for the intra-tag and inter-tag. For intra-tag, the values of mean and standard deviation of cosine similarity are close to unity and small values in thousandths, respectively. For inter-tag, the values of mean and standard deviation of cosine similarity have a decreasing linear trend from tag $\mathrm{A}$ to $\mathrm{C}$. The average values of inter-tag cosine similarity show a well separation ranging from 1 to 0.8991 for tag A to C, respectively, where the worse average intratag cosine similarity is 0.9963 which is insignificant. Therefore, it shows that the imposed variations in the dimension parameters are enough to distinguish between the different tags. In future a larger number of tags can be realized with natural process variations and subsequently a probabilistic model can be presented. Also the radiation measurement in anechoic chamber could be taken into consideration.

TABLE I. MEAN AND STANDARD DEVIATION OF COSINE SIMILARITY.

\begin{tabular}{|c|c|c|c|}
\hline \multirow{2}{*}{ Tag } & \multicolumn{3}{|c|}{ Cosine Similarity } \\
\cline { 2 - 4 } & & Intra-tag & Inter-tag \\
\hline \multirow{2}{*}{$\mathrm{A}$} & mean & 0.9977 & 1 \\
\cline { 2 - 4 } & std & 0.0030 & 0 \\
\hline \multirow{2}{*}{$\mathrm{B}$} & mean & 0.9963 & 0.9490 \\
\cline { 2 - 4 } & std & 0.0035 & 0.0050 \\
\hline \multirow{2}{*}{$\mathrm{C}$} & mean & 0.9967 & 0.8991 \\
\cline { 2 - 4 } & std & 0.0034 & 0.0069 \\
\hline
\end{tabular}

\section{TOWARDS CHIPLESS THZ AUTHENTICATION}

In this conference, we will also present a new device based on dielectric waveguide associated with a 1D diffraction grating, dedicated to authentication of manufactured products in $\mathrm{THz}$ frequency range. One of the applications is for example authentication of integrated circuits (IC). Indeed, the rear side of the silicon substrate of the IC can be engraved with periodic grooves to act as an efficient air-silicon diffraction grating at the top of the bulky silicon substrate. For a given incident angle, when the coupling conditions are verified [10] the reflected $\mathrm{THz}$ spectra exhibits peaks (m-lines) that can be used as a broad band and rich $\mathrm{THz}$ spectral signature to authenticate the IC. The number and the frequency positions of m-lines depend on the geometrical dimensions of the device. So the randomness during the fabrication process can again be exploited to generate unique response in the $\mathrm{THz}$ range. $\mathrm{THz}$ waves are probably the best frequency range to be used to address this application since 1) $\mathrm{THz}$ wavelengths (0.1-1 $\mathrm{mm}$ ) are comparable to typical IC dimensions to be measured, and 2) packaging materials are almost transparent below $1 \mathrm{THz}$, permitting non-contact and non-destructive inspection [11].

\section{A. Device and Method Principle}

The device is composed of a silicon waveguide and a 1D diffraction grating, which rules the coupling of the incoming $\mathrm{THz}$ radiation within the silicon waveguide (see Fig. 4). In such a device, the number of guided modes and their respective frequency position mainly depend on the period $\Lambda$ of the grating, the thickness $e$ of the waveguide, and of course the incidence angle $\Theta$, whereas the depth $p$ of the grooves mainly impacts the coupling efficiency [10]-[12]. Thereby, this device acts as a frequency selective coupler.

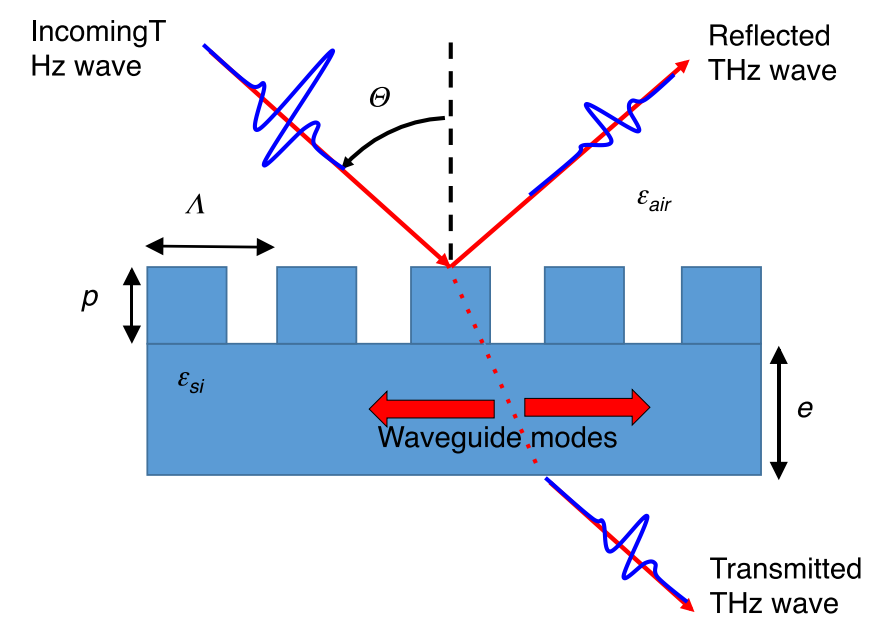

Fig. 4. Principle scheme of a planar dielectic wave guide associated to a $1 \mathrm{D}$ diffraction grating for $\mathrm{THz}$ signature shaping.

In the following simulations, we considered an air-silicon rectangular grating whose permittivities in the considered range $(100 \mathrm{GHz}$ to $1 \mathrm{THz})$ are $\varepsilon_{\text {air }}=1$ and $\varepsilon_{s i}=11.65+j 5.10^{-3}$, respectively. This latter value corresponds to high resistivity silicon $\left(>1000 \Omega . \mathrm{cm}^{-1}\right)$. As an example, we plot Fig. 5, the modulus of the reflection coefficient of the sample presented in Fig. 4, for two values of the grating period: $\Lambda=300 \mu \mathrm{m}$ (red curve) and $350 \mu \mathrm{m}$ (blue curve), whereas the rectangular grooves depth $p=80 \mu \mathrm{m}$, the incident angle $\Theta=10^{\circ}$, and the waveguide thickness $e=220 \mu \mathrm{m}$.

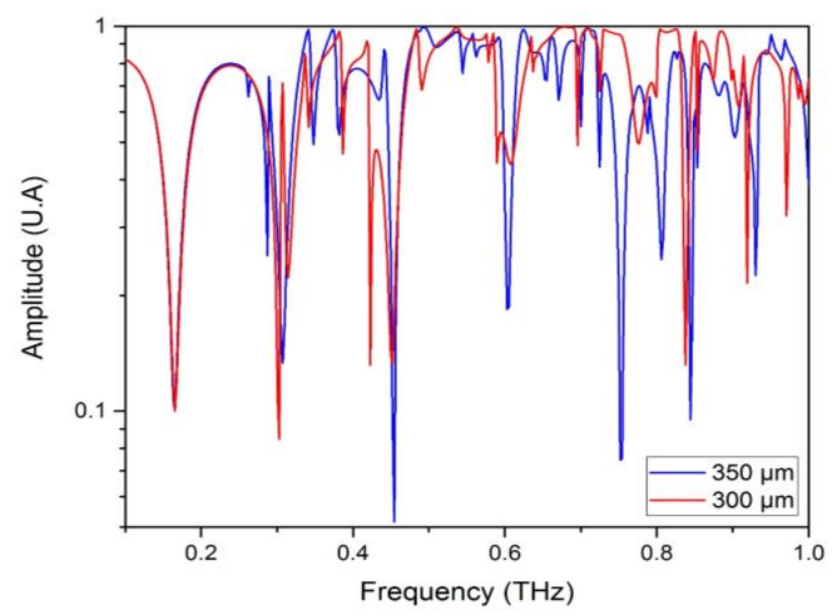

Fig. 5. THz spectral signature (modulus of the reflexion coefficient) of a air-silicon device $(p=80 \mu \mathrm{m}, e=220 \mu \mathrm{m})$.

In the considered frequency range, we can first notice lot of m-lines, corresponding to waves that are coupled into the waveguide with the grating, i.e. waves that verified the coupling conditions [10]. This large number of peaks is the evidence that the signature of such device is very rich in term of spectral information, and can therefore be used for 
authentication. Secondly, comparing the plots obtained for different grating period, it is also obvious that this information can be widely varied by changing the geometrical parameters of the structure.

\section{B. Results}

To definitively prove that the $\mathrm{THz}$ signature of such devices is very sensitive to its geometrical parameters, we studied the impact of both, the grating period and the thickness of the waveguide without changing the grating depth $p$, which has been fixed to $80 \mu \mathrm{m}$. Moreover, in the present study the reflected configuration has been preferred to address authentication of IC whose metallization levels prevent any precise $\mathrm{THz}$ measurements in transmission. Nevertheless, note that similar results can be obtained considering the $\mathrm{THz}$ wave transmitted through the proposed device, regarding the intended application.

Therefore, to point out the sensitivity of the reflected $\mathrm{THz}$ signature relatively to the geometrical parameters, we compare reflection moduli of modified structures (varying grating period and/or waveguide thickness) to the reference device (presenting arbitrarily $\Lambda=300 \mu \mathrm{m}, e=220 \mu \mathrm{m}$ and $p=80 \mu \mathrm{m}$ ), by calculating correlation coefficients over the whole spectral range (between $100 \mathrm{GHz}$ and $1 \mathrm{THz}$ ). To complete the study, and to be able to choose the best way to analyze the results, we compare the variation of the correlation coefficients versus $\Lambda$ and $e$, calculated directly from the reflected signatures, but also on their first and second derivatives.

Results are presented in Figs. 6 and 7, where we vary the grating period from $\Lambda=300$ to $500 \mu \mathrm{m}$ (i.e variation from 0 to $200 \mu \mathrm{m})$, and the waveguide thickness from $p=220$ to $320 \mu \mathrm{m}$, respectively. The incidence angle $\left(\Theta=10^{\circ}\right)$ and the grooves depth $(p=80 \mu \mathrm{m})$ remain constant for all the simulations.

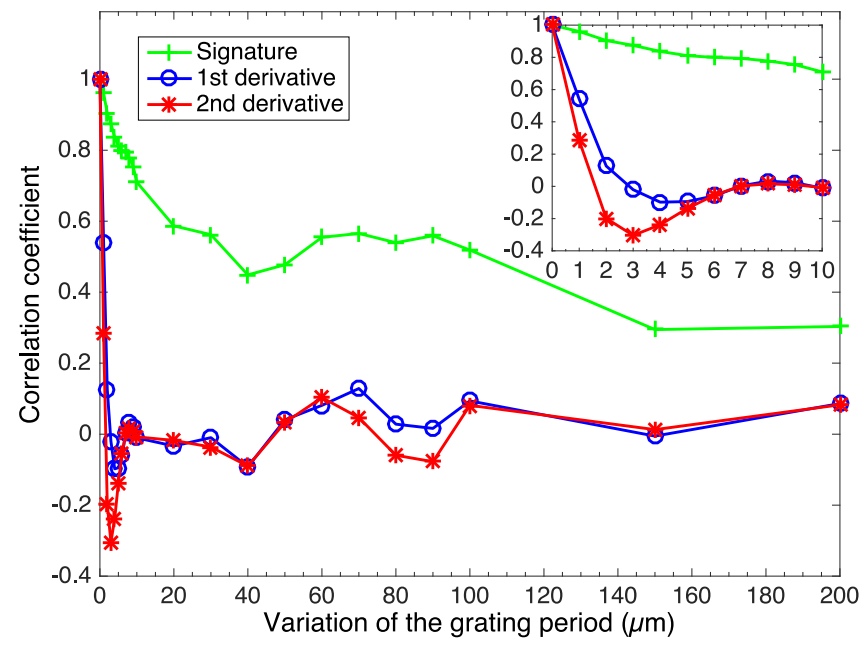

Fig. 6. Correlation coefficient versus the variation of grating period $\Lambda$, calculated on the modulus of the reflexion coefficient and on its first and second derivatives $\left(\Theta=10^{\circ}, e=300 \mu \mathrm{m}, p=80 \mu \mathrm{m}\right)$.

Considering Fig. 6, we notice that the correlation coefficient rapidly drops when $\Lambda$ varies only of a few $\mu \mathrm{m}$, showing it is possible to discriminate two close structures by comparing their $\mathrm{THz}$ signatures in reflection. In addition, the drop is drastically increased when considering the first or even the second derivative of the reflection modulus as it reaches roughly 0 (i.e. $\mathrm{THz}$ signatures have no similarity) for a grating period difference of only $3 \mu \mathrm{m}$ (see insert in Fig. 6). The very same behavior can be noticed in Fig. 7 when the waveguide thickness $e$ varies. Thereby, the proposed structure can be potentially used as authenticator.

More results will be presented during the conference, like for example the effect of the variation of permittivity of the dielectric material constituting the device [13], or the presence of some more dielectric layers [14]. Measurements will be also performed to experimentally validate these first theoretical results.

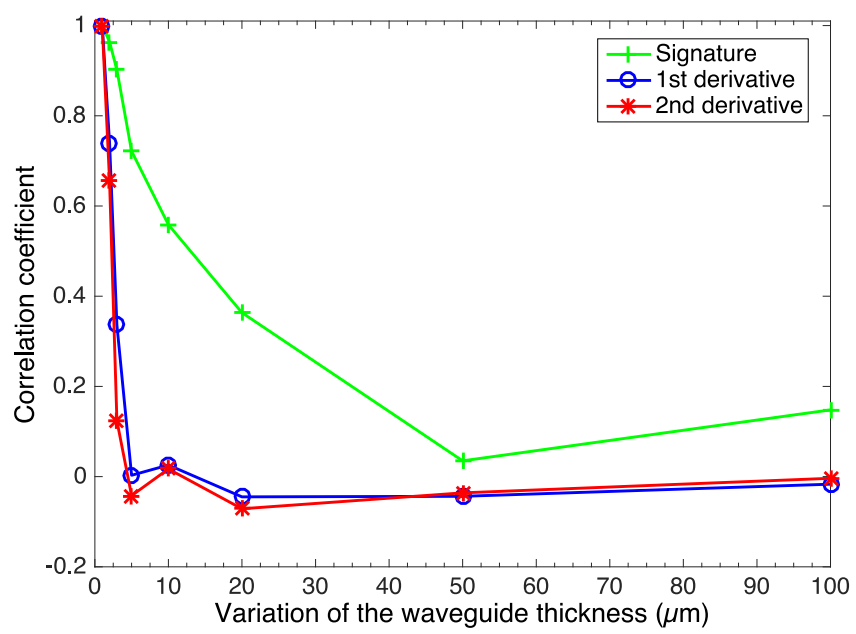

Fig. 7. Correlation coefficient versus the variation of the waveguide thickness $e$, calculated on the modulus of the reflexion coefficient and on its first and second derivatives $\left(\Theta=10^{\circ}, p=80 \mu \mathrm{m}, \Lambda=300 \mu \mathrm{m}\right)$.

\section{CONCLUSION}

In this paper we reported the design of chipless tags operating both in RF and, in $\mathrm{THz}$ domains. We demonstrated the capabilities of chipless technology to be used for authentication applications. The two types of tags exhibit interesting performance.

In the case of the RF solution, three chipless RFID tags were realized by imposing an assumed fabrication tolerance to the geometric dimensions which have a less effect on frequency of resonance. The mean and standard deviation of cosine similarity for the intra-tag and inter-tag were presented. A well separation between the intra-tag and intertag cosine similarity was observed which will be more significant if the variations along $L$ would be taken into account. This proves that geometrical variation not directly link the frequency of resonance of the tag can be used to discriminate tags.

In the case of the THz solution, we can also conclude that because of uncertainties of either the fabrication techniques for the grating (chemical etching or mechanical milling), or the thickness of the device (the wafer thickness can vary of several $\mu \mathrm{m}$ ), it is possible to authenticate the $\mathrm{THz}$ signature 
of such grating/waveguide structure just from the random variations due to manufacturing. Of course, if these geometrical parameters are deliberately modified, the effects will be controlled and even more visible. Secondly, we have shown that the modulus of the $\mathrm{THz}$ wave reflected (or transmitted) by the device is not the best signature to be considered for authentication application as its first and second derivatives exhibit much faster decaying correlation coefficients with variation of geometrical parameters $\Lambda$ and e. Finally, as the materials classically used for the IC packaging is transparent in the considered $\mathrm{THz}$ range, the proposed device can be buried in the packaging without being altered. Therefore the grating is out of sight leading to device robustness against counterfeiting.

\section{ACKNOWLEDGMENT}

The authors would like to acknowledge the University of Grenoble Alpes for financially supporting this project via the AGIR program. The authors are also grateful to the Région Auvergne-Rhône-Alpes for supporting this project via the ARC6 program.

\section{REFERENCES}

[1] E. Perret, A. Vena, S. Tedjini, Y. Boutant, and C. Halope, "Item comprising a barcode with an electromagnetic signature," US20150102105 A1, 16-Apr-2015.

[2] E. Perret, M. Hamdi, G. E. P. Tourtollet, R. Nair, F. Garet, A. Delattre, A. Vena, L. Duvillaret, P. Martinez, S. Tedjini, and Y. Boutant, "THID, the next step of chipless RFID," in IEEE International Conference on RFID, Florida, USA, 2013, pp. 261268.

[3] C. Yang and A. P. Sample, "EM-ID: Tag-less identification of electrical devices via electromagnetic emissions," in IEEE International Conference on RFID, Florida, USA, 2016, pp. 1-8.

[4] K. Yang, D. Forte, and M. M. Tehranipoor, "UCR: An unclonable chipless RFID tag," in IEEE International Symposium on Hardware Oriented Security and Trust (HOST), Washington, USA, 2016, pp. 712.

[5] A. Vena, E. Perret, and S. Tedjini, "Chipless RFID Tag Using Hybrid Coding Technique," IEEE Trans. Microw. Theory Tech., vol. 59, no. 12, pp. 3356-3364, Dec. 2011.

[6] O. Rance, R. Siragusa, P. Lemaitre-Auger, and E. Perret, "Toward RCS Magnitude Level Coding for Chipless RFID," IEEE Trans. Microw. Theory Tech., vol. 64, no. 7, pp. 2315-2325, Jul. 2016.

[7] R. Garg, I. Bahl, and M. Bozzi, Microstrip Lines and Slotlines, Third Edition: Artech House, 2013.

[8] V. Deepu, A. Vena, E. Perret, and S. Tedjini, "New RF identification technology for secure applications," in IEEE International Conference on RFID-Technology and Applications, Guangzhou, China, 2010, pp. 159-163.

[9] A. Vena, E. Perret, and S. Tedjini, Chipless RFID based on RF Encoding Particle: Realization, Coding and Reading System. Elsevier, 2016

[10] J. F. Roux, F. Aquistapace, F. Garet, L. Duvillaret, and J. L. Coutaz, "High efficiency grating coupling of $\mathrm{THz}$ pulse radiation into dielectric waveguide," Electron. Lett., vol. 37, no. 23, pp. 13901392, Nov. 2001.

[11] K. Ahi and M. Anwar, "Advanced terahertz techniques for quality control and counterfeit detection," in Proc. SPIE 9856, Terahertz Physics, Devices, and Systems X: Advanced Applications in Industry and Defense, 2016, p. 98560G.

[12] J.-F. Roux, F. Aquistapace, F. Garet, L. Duvillaret, and J.-L. Coutaz, "Grating-assisted coupling of terahertz waves into a dielectric waveguide studied by terahertz time-domain spectroscopy," Appl. Opt., vol. 41, no. 30, p. 6507, Oct. 2002
[13] F. Aquistapace, L. Duvillaret, F. Garet, J.-F. Roux, and J.-L. Coutaz, "Photovariation of grating-assisted coupling of terahertz waves into a silicon waveguide," J. Appl. Phys., vol. 94, no. 12, pp. 7888-7891, Dec. 2003.

[14] Y. Laamiri, F. Garet, and J.-L. Coutaz, "Thin-film characterization by terahertz time-domain spectroscopy using grating-assisted excitation of guided modes," Appl. Phys. Lett., vol. 94, no. 7, p. 71106, Feb. 2009. 